Swarthmore College

Works

5-1-1996

\title{
Critical Point For The Blue-Phase-III-Isotropic Phase Transition In Chiral Liquid Crystals
}

Z. Kutnjak

C. W. Garland

Colin G. Schatz , '97

Peter J. Collings

Swarthmore College, pcollin1@swarthmore.edu

C. J. Booth

See next page for additional authors

Follow this and additional works at: https://works.swarthmore.edu/fac-physics

Part of the Physics Commons

Let us know how access to these works benefits you

\section{Recommended Citation}

Z. Kutnjak; C. W. Garland; Colin G. Schatz , '97; Peter J. Collings; C. J. Booth; and J. W. Goodby. (1996).

"Critical Point For The Blue-Phase-III-Isotropic Phase Transition In Chiral Liquid Crystals". Physical Review E. Volume 53, Issue 5. 4955-4963. DOI: 10.1103/PhysRevE.53.4955

https://works.swarthmore.edu/fac-physics/158

This work is brought to you for free by Swarthmore College Libraries' Works. It has been accepted for inclusion in Physics \& Astronomy Faculty Works by an authorized administrator of Works. For more information, please contact myworks@swarthmore.edu. 


\section{Authors}

Z. Kutnjak; C. W. Garland; Colin G. Schatz, '97; Peter J. Collings; C. J. Booth; and J. W. Goodby 


\title{
Critical point for the blue-phase-III-isotropic phase transition in chiral liquid crystals
}

\author{
Zdravko Kutnjak and Carl W. Garland \\ School of Science and Center for Material Science and Engineering, Massachusetts Institute of Technology, \\ Cambridge, Massachusetts 02139 \\ Colin G. Schatz and Peter J. Collings \\ Department of Physics and Astronomy, Swarthmore College, Swarthmore, Pennsylvania 19081 \\ Christopher J. Booth and John W. Goodby \\ Department of Chemistry, University of Hull, Hull HU6 7RX, United Kingdom
}

(Received 1 February 1996 )

\begin{abstract}
The highly chiral compound $S, S-4^{\prime \prime}$-(methylbutyl)phenyl-4'-(methylbutyl) biphenyl carboxylate $(S, S$ MBBPC) undergoes a continuous supercritical evolution from the isotropic (I) phase to the third blue phase (BPI III). Mixtures of $S, S$-MBBPC and its racemate have been studied with high-resolution calorimetry capable of quantitative latent heat determinations and with optical activity measurements. Both experiments indicate that the first-order BP III-I transition line ends at a critical point when the chiral mole fraction $X_{c} \simeq 0.45$. Analysis of $C_{p}$ and optical activity data for the near-critical mixture with $X=0.45$ indicates meanfield behavior instead of the theoretically predicted Ising fluctuation behavior, which would be analogous to that at the liquid-gas critical point of a simple fluid. It is speculated that the Ginzburg criterion can explain this mean-field behavior since the critical regime may be too small for experimental observation, as is the case for almost all Smectic- $A-$ Smectic- $C$ transitions. [S1063-651X(96)09605-5]

PACS number(s): 64.70.Md, 65.20.+w, 61.30.-v
\end{abstract}

\section{INTRODUCTION}

Chiral liquid-crystal systems can exhibit a variety of complex "defect" phases not observed in nonchiral or racemic systems. These include three blue phases (BP I, BP II, and BP III) that can occur between the chiral nematic $\left(N^{*}\right)$ and isotropic $(I)$ phases [1] and twist-grain-boundary phases (TGB- $A$ and TGB- $C$ ) that can occur between $N^{*}$ and the smectic Sm- $A$ or $\mathrm{Sm}-C$ phases [2]. Of all these relatively new phases, the amorphous blue phase BP III is the least well understood in terms of structure [3].

Recently it has been shown that the highly chiral compound $S, S-(+)-4^{\prime \prime}$-(methylbutyl)phenyl-4'-(methylbutyl)-4biphenylcarboxylate $\left(S, S\right.$-MBBPC, $\left.M=414 \mathrm{~g} \mathrm{~mol}^{-1}\right)$

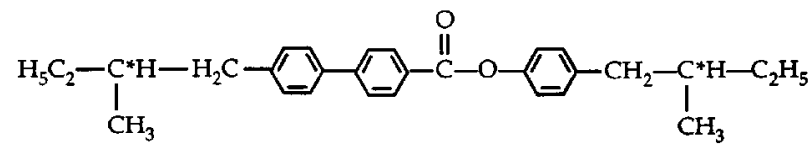

evolves continuously from BP III to $I$ without undergoing a thermodynamic phase transition [4,5]. This observation is consistent with the known trends in previously studied chiral compounds: chirality drives the system toward a more weakly first-order BP III-I transition [5-8]. Specifically, studies of $S$-(+)-4-(2-methylbutyl)phenyl-4-hexyloxybenzoate (CE4) show that the BP III-I latent heat [7] and the discontinuous jump in the static light scattering intensity [5] decrease appreciably with increasing chiral mole fraction in mixtures of a chiral enantiomer with the racemate. Keyes has proposed that coupling between fluctuating chiral modes could cause the first-order BP III-I transition line to terminate in a critical point [9].

The existence of a supercritical BP III to $I$ evolution in $S, S$-MBBPC has an important implication: the BP III and $I$ phases must possess the same macroscopic symmetry. In this case, just like that for the liquid-gas transition in simple fluids, the only possible transition is first order since no symmetry breaking occurs. Naturally, such a first-order coexistence line can terminate in a critical point. Indeed, a recent theory of the BP III-I critical point, inspired in part by the experimental results of Refs. [4] and [5], predicts that this point belongs to the same Ising universality class as the liquid-gas critical point [10]. Two obvious experimental challenges are (a) to characterize the BP III structure (possibly a random isotropic distribution of double twist cylinders, as suggested in [11]) and (b) to characterize the critical behavior of various properties near the BP III-I critical point. The present work tackles the latter issue via calorimetric and optical activity measurements of mixtures of $S, S$-MBBPC with its racemate.

The phase diagram resulting from our study is given in Fig. 1, which also shows previously published data of Yang and Crooker [12]. One difference is the nature of the racemate used. The racemic mixture used by Yang and Crooker contained equal amounts of $S, S$-MBBPC and $R, R$-MBBPC. In the present work, we have used a racemic mixture of all four enantiomers- $S, S-, R, R-, R, S-$, and $S, R$-MBBPCsince the synthesis of this racemate is much simpler. Possibly that difference may explain the slight disagreements in the range $0.6<X<0.8$, where $X$ is the "mole fraction" of the $S, S$-chiral MBBPC. Actually, $X$ represents the number of moles of $S, S$-MBBPC added to $1-X$ moles of the racemic mixture or the number of moles of uncompensated chiral material per mole of MBBPC. In general the agreement is good, especially in the vicinity of $X=0.45$, which represents the critical composition. Polarizing microscope observations for our mixtures in the range $0.35 \leqslant X \leqslant 0.45$ confirm the 


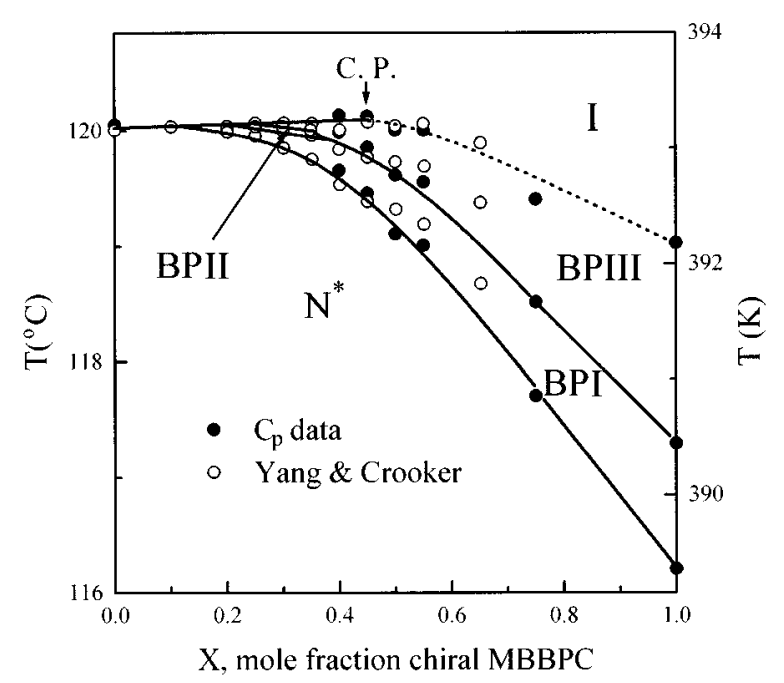

FIG. 1. Phase diagram for mixtures of $S, S$-MBBPC with the racemate. The Yang and Crooker data are from Ref. [12]. The solid lines represent first-order transitions. The dotted line is not a transition line but the locus of maxima in the thermodynamic response functions (in the present case $C_{p}$ ).

calorimetric transition temperatures and show that there is a BP II phase for $X=0.352$ but not for $X \geqslant 0.375$. The phase diagram shown in Fig. 1 agrees well with the universal phase diagram for chiral materials that exhibit blue phases [13] if one keeps in mind that the high chirality of $S, S$-MBBPC means a BP III-I critical point occurs and the BP II phase exists only at rather low $X$ values.

The calorimetric results obtained at the Massachusetts Institute of Technology are presented in Sec. II, while the optical activity data from Swarthmore College are given in Sec. III. The analysis and discussion of these data are given in Sec. IV, which includes a comment on the mean-field nature of our data.

\section{CALORIMETRIC RESULTS}

The $S, S$-MBBPC used in this work was obtained from $\mathrm{BDH}$, a subsidiary of Merck, while the racemic MBBPC was synthesized at the University of Hull. The BDH catalog designation of CE2 is used by some other investigators, but this seems undesirable since CE4 and CE6 are members of a different homologous series and confusions could arise. Care was taken in handling the materials - they were thoroughly degassed by vacuum pumping, the preparation of the mixtures and filling of the cells were carried out in a dry nitrogen atmosphere, and the silver cells were hermetically closed with a cold-weld tin seal. The samples had good thermal stability, and the transition temperatures exhibited very slow drifts with time (typically -15 to $-25 \mathrm{mK} / \mathrm{d}$ ).

The calorimeter is capable of operating in two modes: (a) high-resolution measurements of $C_{p}$ using an ac technique and (b) measurements of enthalpy (thus latent heats) using a nonadiabatic scanning technique. Detailed descriptions of this calorimeter are given in Refs. [14] and [15]. Measurements were made on mixtures with $X=0$ (racemate), $0.40,0.45,0.50,0.55$, and 0.75 to augment the previous study of $X=1.0$ [4]. The investigated temperature range fo-

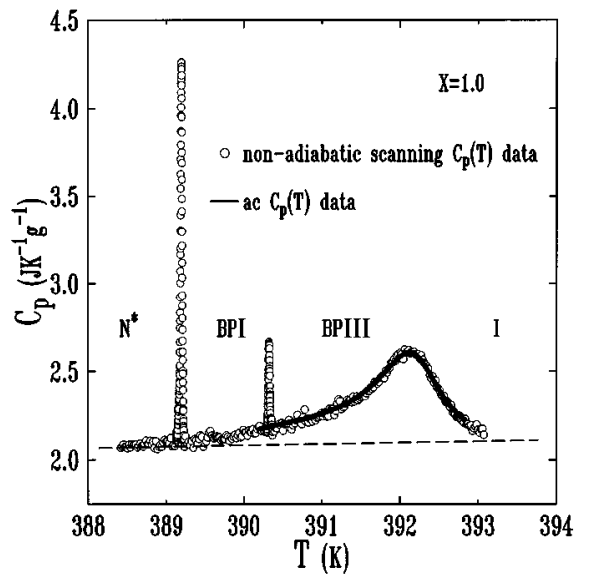

FIG. 2. The specific heat for $S, S$-MBBPC [4]. The nonadiabatic scanning peaks at $T\left(N^{*}-\mathrm{BP}\right.$ I) and $T$ (BP II-BP III) are artificial $C_{p}(\mathrm{eff})$ values related to two-phase coexistence and latent heat effects. Integrating over these peaks yields the latent heat via Eq. (2). The nonadiabatic scan rate was $+4 \mathrm{~K} / \mathrm{h}$, while heating and cooling ac runs at $\omega_{0}=0.196 \mathrm{~s}^{-1}$ were carried out at scan rates of $50 \mathrm{mK} / \mathrm{h}$.

cused on the region of the BP III-I transition or the temperature of supercritical $C_{p}(\max )$; this was typically from $T(\mathrm{BP} \mathrm{III}-I)-4 \mathrm{~K}$ to $T(\mathrm{BP} \mathrm{III}-I)+2.5 \mathrm{~K}$. This region included all three transitions- $T\left(N^{*}-\mathrm{BP}\right.$ I), $T$ (BP I-BP III), $T$ (BP III-I) - observable for chiral mixtures with $X \geqslant 0.375$.

Figure 2 shows the supercritical BP III- $I$ behavior reported for pure $S, S$-MBBPC [4]. The nonadiabatic scanning method measures an effective heat capacity $C_{\text {eff }}$ defined by

$$
C_{\text {eff }}=\left(P-\frac{T-T_{b}}{R}\right) / \frac{d T}{d t},
$$

where $P$ is the dc power input, $T$ is the time-dependent temperature of the sample, $T_{b}$ is the time-independent temperature of the heat bath, and $R$ is the thermal resistance between the sample and bath. Thus $\left(T-T_{b}\right) / R$ represents the rate of heat leak between sample and bath. The latent heat $L$ is then given by

$$
L=\int_{T_{1}}^{T_{2}}\left[C_{\mathrm{eff}}-C_{p}(\operatorname{coex})\right] d T,
$$

where $C_{p}$ (coex) is the heat capacity of the two-phase coexisting mixture in the absence of phase conversion. Thus $C_{p}(\operatorname{coex})$ accounts for the heat effects associated with raising the temperature of a two-phase mixture over the narrow coexistence range from $T_{1}$ to $T_{2}$, and in this system $C_{p}($ coex $)$ is well represented by the ac $C_{p}(T)$ data. The nonadiabatic scanning technique is completely analogous to the wellestablished adiabatic scanning method [16]. The ac calorimetric technique is well known [17] and is capable of yielding precise $C_{p}$ data with very little scatter. The 450 data points obtained for $S, S$-MBBPC are represented in Fig. 2 by the smooth curve. It should be stressed that the ac method does not measure enthalpy or provide any quantitative information on latent heats. Note that the $C_{p}(\mathrm{ac})$ line goes smoothly through $T$ (BP I-BP III) with no visible anomaly. However, characteristic changes in the phase shift $\phi$ between the input ac power $P_{0} \exp [i \omega t]$ and the observed oscillatory 


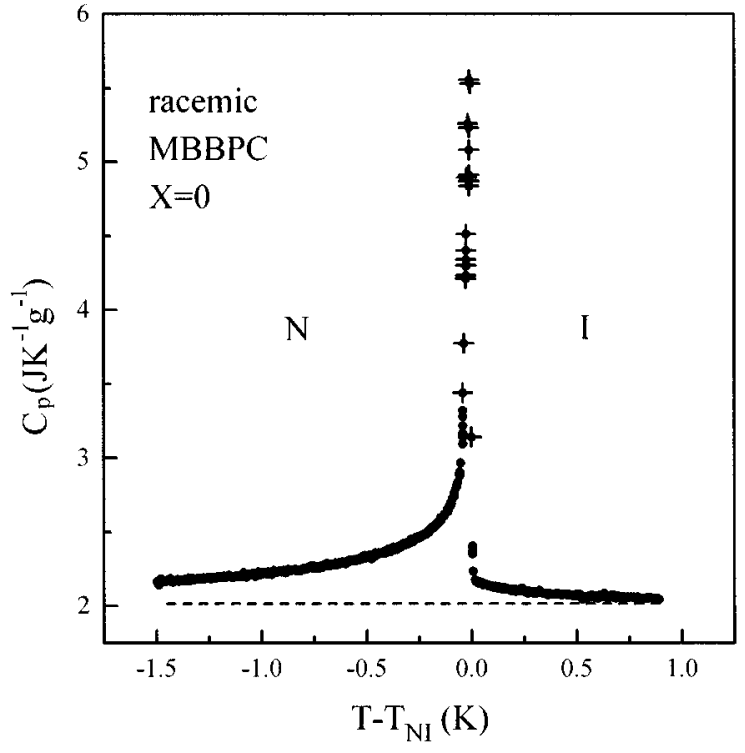

FIG. 3. Specific heat $C_{p}(\mathrm{ac})$ of racemic MBBPC. The + symbols denote data points obtained in a narrow two-phase $N+I$ coexistence region. The first-order transition temperature $T_{N I}$ is 392.98 $\mathrm{K}$, see text for further details.

sample temperature $T_{\mathrm{ac}} \exp [i(\omega t+\phi-\pi / 2)]$ do provide qualitative indications of two-phase coexistence at discontinuous transitions [17]. For $S, S$-MBBPC, these ac phase shift anomalies were seen at the $N^{*}$-BP I and BP I-BP III transitions but were not present in the temperature range 391-393 K, where the BP III-I supercritical evolution occurs. The lack of singular $C_{p}$ behavior at " $T(\mathrm{BP}$ III-I)" and the absence of any excess enthalpy that might represent smeared latent heat are the features indicating supercritical behavior for $S, S$-MBBPC. See Ref. [4] for further discussion.

The heat capacity behavior of the racemic mixture is shown in Fig. 3. In the absence of chirality, there are no blue phases and the $N^{*}$ phase becomes the simple nematic $N$ phase. The dashed line here and in Fig. 2 represents $C_{p}$ (background), the regular specific heat behavior that would occur if no phase transitions took place. As with all $N-I$ transitions, this transition is first order with significant pretransitional fluctuation wings. The integrated enthalpy associated with these wings $\delta H=\int \Delta C_{p} d T$, where $\Delta C_{p}=C_{p}-C_{p}$ (background), is $670 \mathrm{~mJ} \mathrm{~g}^{-1}$. Data points denoted by a plus sign are artificial $C_{p}$ values obtained in a narrow two-phase coexistence region. There are anomalies in the phase shift $\phi$ associated with these points, as is observed at all $N-I$ or $N^{*}-I$ transitions [17].

The specific heat curves obtained by ac calorimetry for five mixtures of $S, S$-MBBPC plus racemic MBBPC are shown in Fig. 4. Only the mixture with $X=0.40$ provided phase shift evidence of first-order two-phase coexistence at $T(\mathrm{BP}$ III $-I)$. As discussed below, we find that $X=0.45$ is very close to the critical composition and mixtures with $X \geqslant 0.50$ are all supercritical. Note the marked decrease in $C_{p}(\max )$ as a function of $X$ from $X_{c} \simeq 0.45$ to $X=1.0$ (the latter is shown in Fig. 2). In the three mixtures with $X=0.50$, 0.55 , and 0.75 , expanded plots show a rounded $C_{p}$ maximum qualitatively like that in Fig. 2. The BP I-BP III and

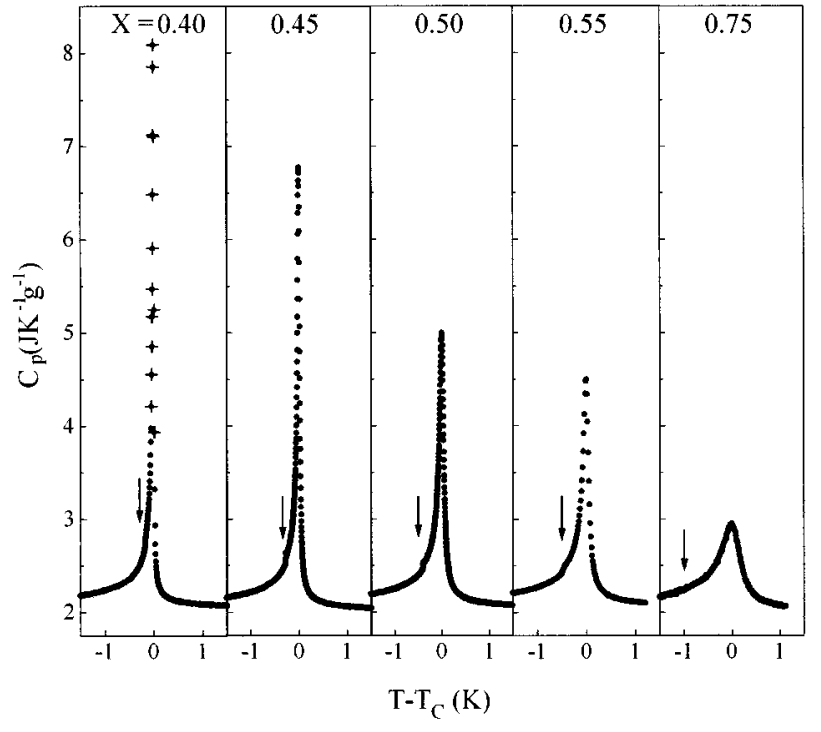

FIG. 4. Detailed view of $C_{p}(\mathrm{ac})$ in the BP III-I region for five mixtures of $S, S$-MBBPC+racemic MBBPC. $X$ is the mole fraction of chiral MBBPC. The vertical arrows indicate the positions of the BP I-BP III transitions. $N^{*}-$ BP I transitions were also observed, but these lie off scale. The + symbols for $X=0.40$ denote data points obtained in a narrow two-phase BP III $+I$ coexistence region. $T_{c}$ is used to denote the transition temperature in all cases, although the transition is first order for $X=0.40$ and supercritical for $X \geqslant 0.50$.

$N^{*}$-BP I (not shown) transitions are all strongly first order, i.e., no pretransitional wings and $100 \%$ of the enthalpy appearing as latent heat $L$. All the data shown in Fig. 4 were obtained at $\omega_{0}=0.196 \mathrm{~s}^{-1}$. In order to test for possible dynamical effects (critical slowing down), measurements were also made at $2 \omega_{0}, \omega_{0} / 3$, and $\omega_{0} / 9$ for the mixtures with $X=0.45$ and 0.50 . The $C_{p}(\omega)$ values agreed for $\omega \leqslant \omega_{0}$ at all temperatures and the $C_{p}\left(2 \omega_{0}\right)$ data deviated only by lying slightly lower at temperatures very close to that for the maximum in $C_{p}$. Thus we feel confident that the $C_{p}\left(\omega_{0}\right)$ data in Fig. 4 represent static heat capacities for $X \geqslant 0.45$.

Nonadiabatic scanning data for mixtures with $X=0.40$ and 0.45 are presented in Fig. 5 together with smooth curves representing the $C_{p}(\mathrm{ac})$ data from Fig. 4. This figure should be compared to Fig. 2, which gives a comparable presentation of data for $S, S$-MBBPC $(X=1.0)$. As expected from the qualitative ac phase shift and frequency dependence information, there is more enthalpy associated with the $X=0.40$ BP III- $I$ transition than indicated by the artificial $C_{p}($ ac) data close to the transition temperature. The fact that $C_{\text {eff }}$ (nonadiabatic) $>C_{p}(\mathrm{ac})$ near $T(\mathrm{BP}$ III $-I)$ is a second and quantitative indication of first-order character. No such excess occurs for the BP III $-I$ transition when $X=0.45$ or 0.50 . Thus the critical composition $X_{c}$ is 0.45 or some smaller value closer to 0.45 than 0.40 . A power-law analysis given in Sec. IV indicates these $X=0.45$ data are nearly singular, which means that $X_{c}$ must be quite close to 0.45 . As in Fig. 2 , there are nonadiabatic scanning peaks at $T\left(N^{*}-\mathrm{BP}\right.$ I $)$ and $T$ (BP I-BP III) and these allow us to determine the latent heats $L$ at these two first-order transitions.

The transition temperatures and enthalpies (where known) for all three observed transitions are given in Table I for each 


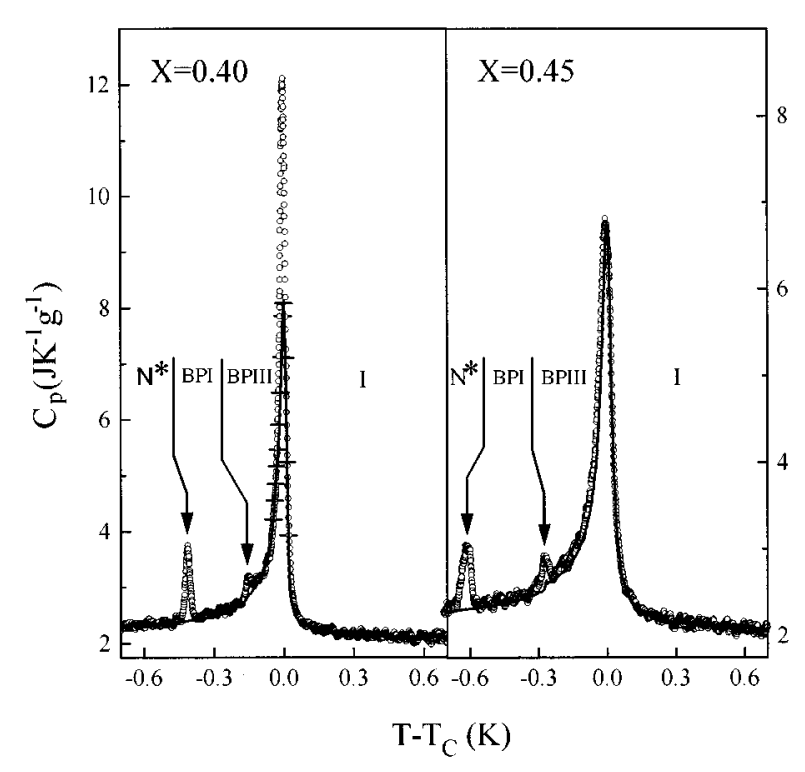

FIG. 5. Specific heat of two MBBPC mixtures with chiral mole fraction $X=0.40$ and 0.45 . The open circles denote nonadiabatic scanning data, and the solid lines represent smooth curves through the $C_{p}(\mathrm{ac})$ data shown in Fig. 4. The mixture with $X=0.40$ has a first-order BP III-I transition, as indicated by $C_{\text {eff }}$ (nonadiabatic) $>C_{p}($ ac) near the transition and by the observation of ac phase shift anomalies over the $C_{p}(\mathrm{ac})$ range denoted by + signs. Note the good agreement between ac and nonadiabatic scanning data at the BP III- $I$ peak for $X=0.45 \simeq X_{c}$.

investigated chiral mixture. Hysteresis in transition temperatures was observed for the first-order $N^{*}-\mathrm{BP}$ I and BP IBP II transitions: typical values were $0.45 \mathrm{~K}$ for $N^{*}-\mathrm{BP}$ I and $0.075 \mathrm{~K}$ for BP I-BP III. A hysteresis of $\sim 0.01 \mathrm{~K}$ was also observed for the BP III $-I$ transition in the $X=0.40$ mixture. The $N^{*}$-BP I and BP I-BP III latent heats are typical of those reported for other chiral liquid crystals. The $N^{*}$-BP I latent heat is $34 \mathrm{~mJ} \mathrm{~g}^{-1}$ for cholesteryl nonanoate (CN) [18] and $87 \mathrm{~mJ} \mathrm{~g}^{-1}$ for CE6 [7]. The sum of latent heats for BP I-BP II and BP II-BP III is $14.6 \mathrm{~mJ} \mathrm{~g}^{-1}$ for CN [18] and $71 \mathrm{~mJ} \mathrm{~g}^{-1}$ for CE6 [7]. These values can be compared to our $L$ values for BP I-BP III. For the BP III- $I$ transition, all prior reports were for first-order transitions but there is both a discontinuous latent $L$ and an integrated en- thalpy $\delta H$ corresponding to the area under the pretransitional $C_{p}$ wings. The corresponding values in $\mathrm{mJ} \mathrm{g}^{-1}$ are $L \simeq 323$ \pm 50 and $\delta H \simeq 860 \pm 50$ for $\mathrm{CN}$ [18], $L=785$ and $\delta H=785$ and $\delta H=1675$ for CE6 [7], and $L \simeq 100$ and $\delta H=986$ for CE4 [7]. The CE4 latent heat is the smallest BP III-I value previously reported.

\section{OPTICAL ACTIVITY}

Optical activity is the rotation of linearly polarized light as it passes through a substance. If the direction of polarization is rotated by an angle $\Phi$ as it passes through a sample of thickness $d$, then the optical activity is simply $\Phi / d$. The experimental apparatus consisted of a laser, fixed polarizer, Faraday modulator, temperature-controlled sample chamber, rotatable polarizer, and a solid-state detector. The rotatable polarizer was controllable in steps of $0.01^{\circ}$ by a computer, and lock-in detection was used to amplify the signal and reduce noise. The computer scanned across the angle of minimum detector output and performed a linear interpolation in order to measure the rotation introduced by the sample with a precision of $\pm 0.001^{\circ}$. The sample of liquid crystal was held between two pieces of glass separated by a 125- $\mu \mathrm{m}$ mylar spacer, sitting in an Instec $\mathrm{mK}-1$ hot stage. Temperature regulation was $\pm 1 \mathrm{mK}$ and optical activity measurements were made at intervals as small as $5 \mathrm{mK}$. All measurements were made upon heating using 633-nm light.

The results of optical activity measurements on three samples are shown in Fig. 6. Notice that in each case the BP I-BP III transition is clearly evident as a discontinuous jump in $\Phi / d$, with no obvious sign of pretransitional effects, but that a discontinuous transition from BP III to the isotropic phase is only evident in the mixture with a chiral fraction of 0.375 . It should be noted that optical activity is not an ideal probe for the presence of a small first-order discontinuity, as indicated by optical activity and light scattering measurements on chiral CE4 [5], which undergoes a very weak first-order BP III-I transition. However, the present optical activity data are fully consistent with the calorimetric data that indicate a critical point at $X_{c} \simeq 0.45$. Figure 6(d) shows a detailed view of the data for all three samples. The quantity $T_{c}$ in this figure corresponds to $T$ (BP III-I) for $X=0.375$, the value of $T_{c}$ obtained from the best fit of $\Phi / d$ data for $X=0.45$ (see Sec. IV), and $T\left(C_{p \max }\right)$ for $X=1$. Although

TABLE I. Transition temperatures (K) and enthalpies for $N^{*}$-BP I, BP I-BP II and BP III-I transitions in mixtures of MBBPC with chiral mole fraction $X$. For $X>X_{c}=0.45$, BP III- $I$ represents a supercritical evolution. $L$ represents the latent heat at a first-order transition and $\delta H$ denotes the integrated enthalpy $\int \Delta C_{p} d T$, where $\Delta C_{p}=C_{p}-C_{p}$ (background). Both $L$ and $\delta H$ are in $\mathrm{mJ} \mathrm{g}^{-1}$.

\begin{tabular}{|c|c|c|c|c|c|c|}
\hline \multirow[b]{2}{*}{$X$} & \multicolumn{2}{|c|}{$N^{*}-\mathrm{BP}$ I } & \multicolumn{2}{|c|}{ BP I-BP II } & \multicolumn{2}{|c|}{ BP III-I } \\
\hline & $T$ & $L$ & $T$ & $L$ & $T$ & Enthalpy \\
\hline 0.40 & 392.64 & 33.9 & 392.90 & 8.2 & 393.06 & $\left\{\begin{array}{c}L \simeq 150 \pm 50 \\
\delta H \simeq 970\end{array}\right.$ \\
\hline 0.45 & 392.435 & 35.4 & 39279 & 9.7 & 393.05 & $\delta H=1120$ \\
\hline 0.50 & 392.01 & 44.0 & 392.53 & 10.5 & 392.93 & $\delta H=1080$ \\
\hline 0.55 & 391.87 & & 392.47 & & 392.28 & $\delta H=1000$ \\
\hline 0.75 & 390.55 & & 391.43 & & 391.60 & $\delta H=875$ \\
\hline 1.0 & 389.20 & 56.4 & 390.34 & 11.3 & 392.12 & $\delta H=830$ \\
\hline
\end{tabular}



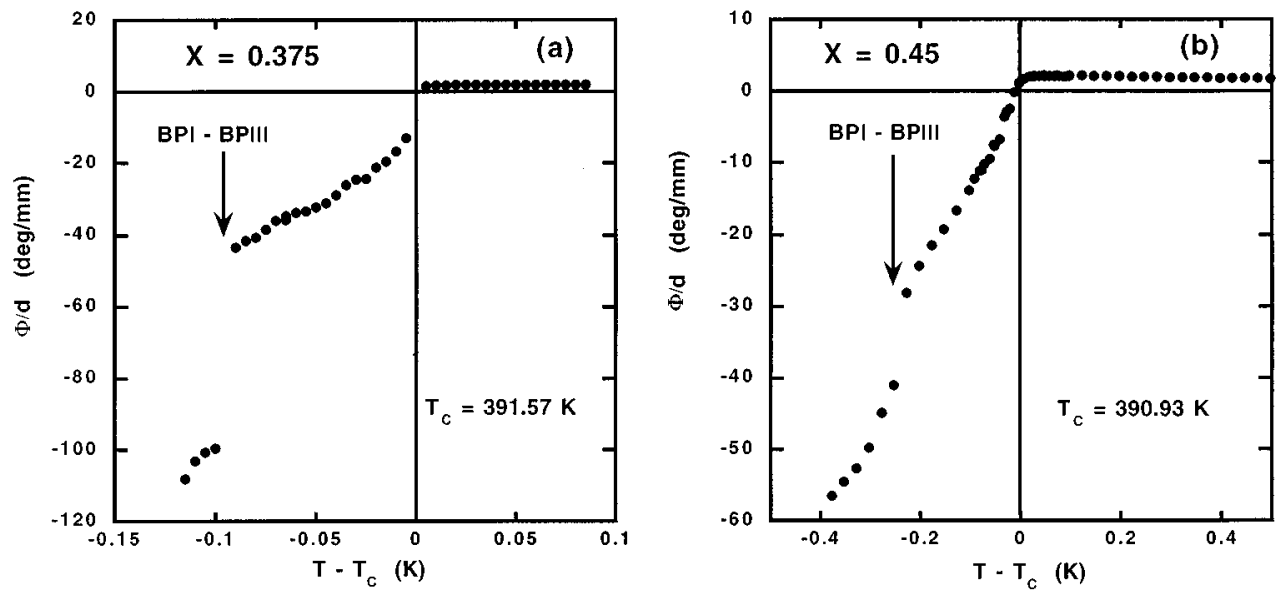

FIG. 6. Optical activity $\Phi / d$ near the BP III-I "transition'" for $S, S$-MBBPC and two mixtures with $X=0.375$ and $X=0.45 \simeq X_{c}$. A discontinuous change is observed at the first-order $N^{*}$-BP I transitions (not shown), the BP I-
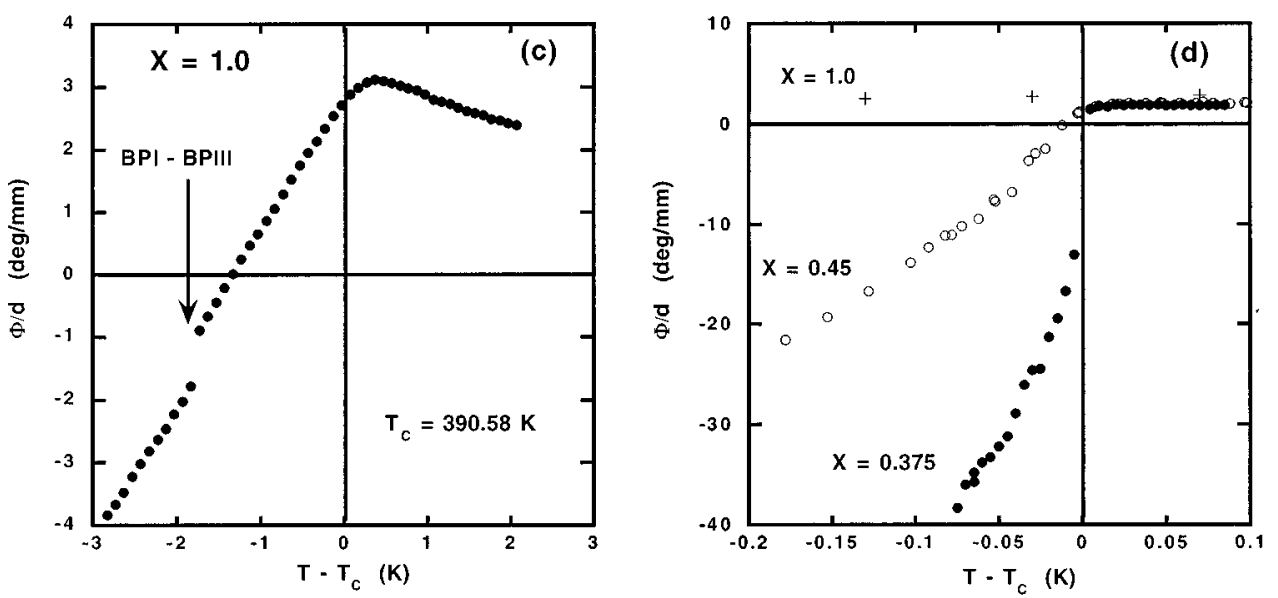
BP III transitions, and the BP III$I$ transition for $X=0.375$. A detailed view of the data very close to $T_{c}$ is given in part (d). The uncertainties in the optical activity are $\pm 1.5, \pm 1.0$, and $\pm 0.5 \mathrm{deg} / \mathrm{mm}$ for $X=0.375,0.45$, and 1.0 , respectively.

the temperatures of the various transitions as measured by heat capacity and optical activity differ by $1.6-2.1 \mathrm{~K}$, the temperature ranges of the various phases consistently agreed to within $0.1 \mathrm{~K}$. Therefore, a systematic shift of a little less than $2 \mathrm{~K}$ can be used in all comparisons between the heat capacity and optical activity results.

All $\Phi / d$ measurements show a temperature dependence in the isotropic phase typical of the pretransitional fluctuations seen in chiral systems. In the $X=0.375$ and 0.45 mixtures, the data in the isotropic phase are dominated by a $\left(T-T^{*}\right)^{-1 / 2}$ dependence over most of the temperature range (see Sec. IV B). In the pure chiral compound, this temperature dependence breaks down at a temperature above the supercritical "transition" temperature as determined from the location of $C_{p}(\max )$ and, of course, above the maximum in $\Phi / d$. This is in agreement with previous optical activity measurements on mixtures of $S, S$-MBBPC with a nematic liquid-crystal compound [19].

\section{ANALYSIS AND DISCUSSION}

This section is concerned with an analysis of the critical behavior of $C_{p}$ and $\Phi / d$ for the $S, S$-MBBPC+racemate mixture with $X=0.45$ and with a discussion of the resulting mean-field character of the BP III-I critical point.

\section{A. Heat capacity analysis}

The heat capacity data for $X=0.45$ have been analyzed over the range of reduced temperatures $\tau=\left(T-T_{c}\right) / T_{c}$ from $\tau=-6.8 \times 10^{-4}$ to $+6.6 \times 10^{-3}$. The limiting factor on the low-temperature side is the BP I-BP III transition, which occurs $0.27 \mathrm{~K}$ below $T_{c}$. For reasons explained below, we have excluded from the fit data over the range from 392.965 to $393.145 \mathrm{~K}$. Thus the minimum $\tau$ values are $\pm 2.3 \times 10^{-4}$. The data analyzed were obtained with the ac technique at a frequency $\omega_{0}=0.196 \mathrm{~s}^{-1}$ since runs at lower frequencies showed no frequency dependence for $C_{p}(\mathrm{ac})$ values. Thus we believe the present values represent the static thermodynamic heat capacity.

Fits of $C_{p}$ were based on the usual renormalization power-law form,

$$
\begin{aligned}
C_{p}= & A^{ \pm}|\tau|^{-x}\left(1+D_{1}^{ \pm}|\tau|^{\Delta_{1}}+D_{2}^{ \pm}|\tau|^{2 \Delta_{1}}+\cdots\right)+B \\
& +E\left(T-T_{c}\right),
\end{aligned}
$$

where the correction-to-scaling exponent $\Delta_{1} \simeq 0.5$, and the exponent $x$ is dependent on the path of approach to the critical point. In the present case, $x$ is not the usual heat capacity exponent $\alpha$. For fitting convenience, Eq. (3) can be rewritten as

$$
C_{p}=A_{1}^{ \pm}|\tau|^{-x}+A_{2}^{ \pm}|\tau|^{-y}+B^{ \pm}+E\left(T-T_{c}\right),
$$

where $y=x-\Delta_{1}$ and $A_{2}^{ \pm}=A^{ \pm} D_{1}^{ \pm}$. In some fits $y$ will be treated as an effective exponent and allowed to vary independently of $x$. Note also that we permit $B^{+} \neq B^{-}$, since if $x=1$ and $2 \Delta_{1}=1$ one obtains $B^{ \pm}=B+A^{ \pm} D_{2}^{ \pm}$. The linear 
TABLE II. Least-squares values of the $C_{p}$ fitting parameters appearing in Eq. (4) for the near-critical mixture with $X=0.45$. Quantities held fixed during a fit are enclosed in square brackets. Range $A$ (108 points) has $\tau_{\max }^{+}=6.75 \times 10^{-4}$ and range $B$ (367 points) has $\tau_{\max }^{+}=6.63 \times 10^{-3}$. For all fits, $\tau_{\max }^{-}=-6.78 \times 10^{-4}$ and $\tau_{\min }^{+}=2.3 \times 10^{-4}$. The units of $A_{1}^{ \pm}, A_{2}^{ \pm}, B^{+}$, and $\Delta B \equiv B^{+}-B^{-}$are $\mathrm{J} \mathrm{K}^{-1} \mathrm{~g}^{-1} ; E$ was held fixed at zero. The $95 \%$ confidence limiting value of $F=\chi_{\nu}^{2}$ (fit $\left.n\right) / \chi_{\nu}^{2}$ (fit $m$ ) is 1.37 for range $A$ and 1.19 for range $B$.

\begin{tabular}{lccccccccccc}
\hline \hline Fit & Range & $T_{c}(\mathrm{~K})$ & $x$ & $y$ & $10^{4} A_{1}^{+}$ & $A_{1}^{-} / A_{1}^{+}$ & $10^{4} A_{2}^{+}$ & $A_{2}^{-} / A_{2}^{+}$ & $B^{+}$ & $\Delta B$ & $\chi_{\nu}^{2}$ \\
\hline 1 & $A$ & 393.053 & 0.997 & & 0.959 & 2.78 & {$[0]$} & {$[1]$} & 2.031 & -0.195 & 2.37 \\
1 & $B$ & 393.049 & 0.970 & & 1.304 & 2.45 & {$[0]$} & {$[1]$} & 2.017 & -0.208 & 2.44 \\
2 & $A$ & 393.049 & 0.983 & 0.447 & 1.074 & 2.60 & 1.2 & 6.78 & 2.030 & -0.191 & 2.21 \\
2 & $B$ & 393.051 & 0.971 & 0.512 & 1.111 & 2.55 & 7.29 & 4.22 & 2.008 & -0.128 & 1.96 \\
3 & $A$ & 393.053 & {$[1]$} & {$[0.5]$} & 0.972 & 2.62 & 0.5 & 92.3 & 2.023 & -0.198 & 2.67 \\
3 & $B$ & 393.052 & {$[1]$} & {$[0.5]$} & 0.867 & 2.58 & 9.00 & 3.71 & 2.008 & -0.139 & 2.10 \\
4 & $B$ & 393.052 & {$[1.241]$} & 0.693 & 0.098 & 1.37 & 4.31 & 5.48 & 2.018 & -0.103 & 4.31 \\
5 & $B$ & 393.048 & {$[0.7925]$} & 0.989 & 4.948 & 3.09 & 0.08 & 2.08 & 2.002 & -0.069 & 3.41 \\
\hline \hline
\end{tabular}

term with slope $E$ arises from the regular (noncritical) contributions to $C_{p}$, while $B=B_{r}+B_{c}$ represents a combination of both regular and critical contributions. The value of $E$ will be fixed at zero for all the fits given in Table II, since this is consistent with a $C_{p}$ (background) line determined from $C_{p}$ data over a wide temperature range.

Table II shows fits over two ranges; fits with the same arabic number are of the same type (i.e., fits $n A$ and $n B$ differ only in the range of data not the nature of parameters held fixed). Simple power-law fits $\left(A_{2}^{ \pm}=0\right.$ and $\left.B^{+}=B^{-}\right)$are of very poor quality, $\chi_{\nu}^{2}=15$, but do indicate that the leading exponent $x$ must be large since they yield $x \simeq 0$.8. Significantly improved fits are obtained by either allowing $B^{+} \neq B^{-}$ (fits $1 A$ and $1 B$ ) or allowing both $B^{+} \neq B^{-}$and nonzero $A_{2}^{ \pm}$ (fits $2 A, 2 B$ and $3 A, 3 B$ ). The conclusion from these fits is $x=1.0 \pm 0.03$ and $y=0.5 \pm 0.05$. Single-sided fits of the data above $T_{c}$ and below $T_{c}$ separately with a simple power law confirm that $x \simeq 1$ and $\Delta B$ is negative. When more data points near $T_{c}\left(\tau_{\min }^{-}=-8.4 \times 10^{-5}\right.$ and $\tau_{\min }^{+}=4.05 \times 10^{-5}$ or $\left.3.57 \times 10^{-5}\right)$ are included in the fit, larger $\chi_{\nu}^{2}$ values are obtained due to systematic instrumental deviations but the values of the fitting parameters hardly change at all (e.g., $x=1.0$ $\pm 0.03, y=0.5 \pm 0.08, \Delta B=-0.10$ to -0.15 for range $B)$.

Since there is a theoretical possibility [10] that fluctuations of Ising universality dominate at this BP III-I critical point and $x$ can be either $\gamma$ or $\gamma / \Delta$ (where $\Delta=\beta+\gamma$ is the gap exponent), we have tested fits with $x=[1.241]$ and $x=[0.7925]$. As seen by the $\chi_{\nu}^{2}$ values for fits $4 B$ and $5 B$, such exponent values can be rejected with $95 \%$ confidence based on the $F$ test.

The agreement between $C_{p}$ data for the $X=0.45$ mixture and fit $3 B$ is shown in Fig. 7. Part (b) of this figure presents a deviation plot of $\Delta C_{p}=C_{p}(\mathrm{obs})-C_{p}$ (fit). Although these deviations are quite random in character for $|\tau|>2.3 \times 10^{-4}$, there are large systematic deviations for temperatures closer to $T_{c}\left(\left|T-T_{c}\right|<90 \mathrm{mK}\right)$. These do not represent some theoretical crossover in the true $C_{p}$ behavior but rather reflect instrumental effects related to the large value of the exponent $x$ and the relatively large $\Delta T_{\text {ac }}$ amplitude used. In order to ensure a very good signal to noise ratio, we used an ac heater power which resulted in $\Delta T_{\mathrm{ac}} \simeq 17 \mathrm{mK}$. For typical heat capacity behavior characterized by a critical exponent $\alpha$ in the range $\alpha_{X Y}=-0.007$ to $\alpha_{\text {eff }} \simeq+0.5$, this $\Delta T_{\text {ac }}$ value would cause instrumental distortions for $\left|T-T_{c}\right| \leqslant 20 \mathrm{mK}$. However, for the present case with the surprisingly large expo- nent value of $x \simeq 1.0$, the instrumental distortion is much larger. The smooth curves given in Fig. 7(b) were obtained from the convolution of a theoretical curve based on the parameters of fit $3 B$ but with a truncation over the range $T_{c}-20 \mathrm{mK}$ to $T_{c}+7 \mathrm{mK}$, which reproduced the observed $C_{p}(\max )$ value at $T_{c}$.

\section{B. Optical activity analysis}

The optical activity $\Phi / d$ data for the mixture with $X=0.45$ have been analyzed over the reduced temperature

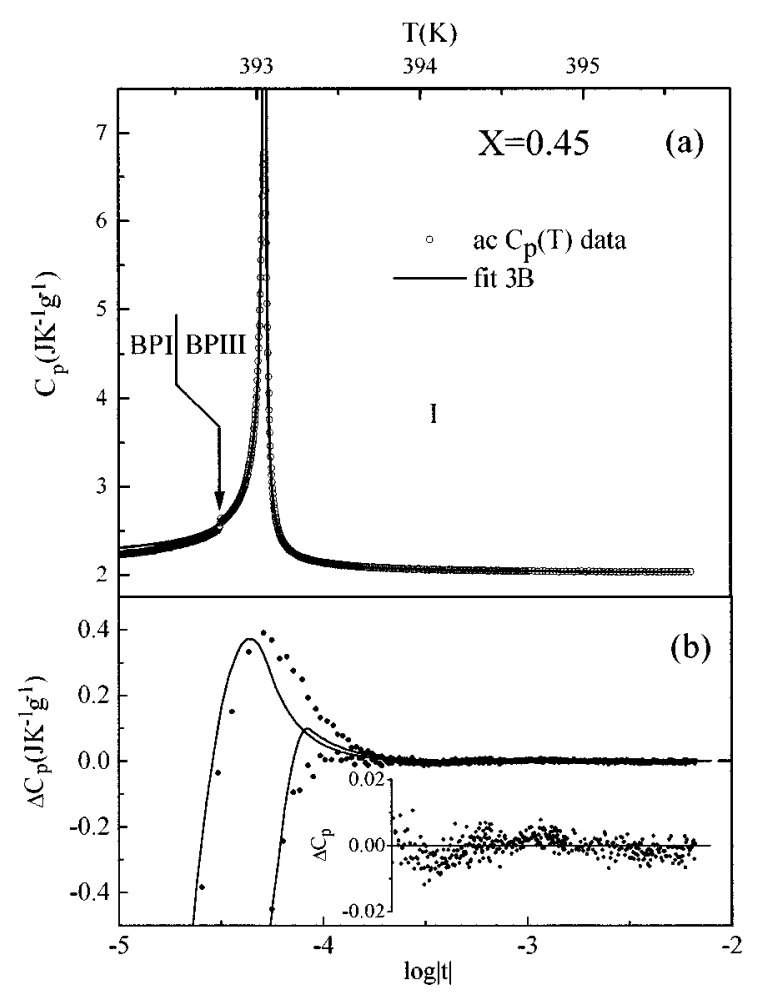

FIG. 7. Comparison of $C_{p}$ data and theoretical fit curve for the near-critical mixture with $X=0.45$. (a) The smooth curve represents fit $3 B$ in Table II for which the critical exponent $x=1.0$. Note that the latent heat at the first-order BP I-BP III transition is not observed with ac calorimetry. (b) Deviations $\Delta C_{p}=C_{p}(\mathrm{obs})-C_{p}$ (fit). The smooth curves in this part represent the result of generating " $C_{p}$ (obs)" values by a convolution of the theoretical form that has been truncated over the narrow range $|\tau|<6 \times 10^{-5}$. 
TABLE III. Least-squares values of the $(\Phi / d)$ optical activity fitting parameters for representing the near-critical mixture $X=0.45$ with Eq. (9). Quantities held fixed during a fit are enclosed in square brackets. There are 50 data points in the fitting range $\left(\tau\right.$ from $-3.25 \times 10^{-4}$ to $\left.+1.53 \times 10^{-3}\right)$. The units of $A_{1}^{+}, A_{2}^{+}$, and $B$ are deg $\mathrm{mm}^{-1}$; those of $C$ are $\operatorname{deg} \mathrm{mm}^{-1} \mathrm{~K}^{1 / 2}$.

\begin{tabular}{lcccccccc}
\hline \hline Fit & $T_{c}(\mathrm{~K})$ & $z$ & $A_{1}^{+}$ & $A_{1}^{-} / A_{1}^{+}$ & $A_{2}^{+}$ & $A_{2}^{-} / A_{2}^{+}$ & $B$ & $C$ \\
\hline 1 & 390.928 & 0.77 & 64.5 & -149 & {$[0]$} & {$[1]$} & 2.31 & 1.19 \\
2 & 390.928 & 0.52 & 10.1 & -44.4 & 319 & -197 & 2.36 & 1.43 \\
3 & 390.928 & {$[0.5]$} & 8.55 & -35.7 & 279 & -177 & 2.34 & 1.42 \\
\hline \hline
\end{tabular}

range from $\tau=-3.25 \times 10^{-4}$ to $\tau=+1.53 \times 10^{-3}$. The fit is based on the theoretical connection between $\Phi / d$ and the quantity $\varphi=\psi-\psi_{c}$ :

$$
(\Phi / d)=(\Phi / d)_{c}+a\langle\varphi\rangle+b\langle\varphi\rangle^{2},
$$

where the subscript $c$ represents values at the critical point; see Eqs. (33)-(35) in Ref. [10]. For a path of constant chirality $\kappa=4 \pi / P$ (where $P$ is the pitch for the liquid crystal), the coefficients $a$ and $b$ can be taken to be constants. The order parameter $\langle\psi\rangle$ for chiral isotropic and BP III phases is given by $\langle\psi\rangle=\langle(\nabla \times Q) \cdot Q\rangle=\left\langle\varepsilon_{i j k} Q_{i l} \nabla_{j} Q_{k l}\right\rangle$, where $Q$ is the alignment tensor characterizing the complex spatial pattern in such phases [10]. In the vicinity of the critical point, the order parameter $\langle\varphi\rangle$ obeys the usual scaling relation

$$
\langle\varphi\rangle=|t|^{\beta} F\left(h /|t|^{\Delta}\right),
$$

where $t$ and $h$ are theoretical scaling fields. The critical exponent describing the dependence of $\langle\varphi\rangle$ on the experimental reduced temperature $|\tau|$ at constant $X$ (i.e., constant composition) depends on the path of approach.

In order to proceed, it is necessary to note that there is also a "background" variation for $\Phi / d$ that is not due to $\langle\varphi\rangle$ order parameter variations but arises from a susceptibilitylike term [20]. Thus $(\Phi / d)_{c}$ must be replaced by a temperaturedependent contribution, to be denoted $F_{\text {background }}(T)$, which causes $\Phi / d$ to decrease slowly on heating and is the dominant term above $T_{c}$. Such behavior has been observed in $S, S$-MBBPC and mixtures of $S, S$-MBBPC $+\overline{7} S 5$ (nonchiral 4- $n$-pentylbenzenethio- 4 ' $-n$-heptyloxybenzoate), and the form of $F_{\text {background }}$ is given by [19]

$$
F_{\text {background }}(T)=\frac{\text { const }}{\left(T-T^{*}\right)^{0.5}},
$$

where $T^{*}$ is an empirical parameter with a value smaller than the BP III-I transition temperature. Thus we write

$$
\begin{gathered}
(\Phi / d)=a\langle\varphi\rangle+b\langle\varphi\rangle^{2}+F_{\text {background }}(T), \\
(\Phi / d)=A_{1}^{ \pm}|\tau|^{z}+A_{2}^{ \pm}|\tau|^{2 z}+\frac{C}{\left[T-T_{c^{-}}\left(C^{2} / B^{2}\right)\right]^{0.5}},
\end{gathered}
$$

where the temperature dependence of $\langle\varphi\rangle$ is represented by $\mid \tau^{z}$, with $z$ as an effective critical exponent. By choosing $T^{*} \equiv T_{c}-\left(C^{2} / B^{2}\right)$, the fitting parameter $B$ represents $(\Phi / d)_{c}$, the value of the optical activity at the critical point.

Table III summarizes fits to the ( $\Phi / d)$ data with Eq. (9). A single power-law fit $\left(A_{2}^{ \pm}=0\right)$ yields an effective exponent $z=0.77$, which is difficult to interpret theoretically (see Sec.
IV C). However, fits in which both the $\langle\varphi\rangle$ and $\langle\varphi\rangle^{2}$ terms contribute to $\Phi / d$ are equally good and yield $z \simeq 0$.5. Figure 8 shows the agreement between the $\Phi / d$ data for the nearcritical mixture with $X=0.45$ and fit 2 from Table III. It should be noted that the value of the effective exponent $z$ is not very sensitive to the choice of $F_{\text {background }}$. For example, a single power-law fit with a linear background contribution exhibits systematic deviations for $T>T_{c}$ but yields $z=0.71$.

\section{Discussion}

In order to relate our empirical exponents $x=1.0$ and $z=0.5$ with standard critical exponents, it is necessary to consider the path of approach to the critical point in $(h, t)$ space corresponding to the experimental path $X=0.45 \simeq X_{c}$. Lubensky and Stark [10] give $t=a_{1} \tau+b_{1} \Delta \kappa$ and $h=a_{2} \tau+b_{2} \Delta \kappa$, where $\Delta \kappa=\kappa-\kappa_{c}$. Although it is known that the chirality $\kappa$ varies slightly with $T$ at constant $X$, this variation is much weaker than the dependence of $\kappa$ on $X$. Thus we write

$$
\begin{aligned}
& t=a_{1} \tau+c_{1} \Delta X, \\
& h=a_{2} \tau+c_{2} \Delta X,
\end{aligned}
$$

where $\Delta X=X-X_{c}$. There are two limiting regions in $(h, t)$ space: (1) $|h| \gg|t|^{\Delta}$ and (2) $|h| \ll|t|^{\Delta}$, where the gap exponent $\Delta=\beta+\gamma>1$. A third possibility is a curved path in $(h, t)$

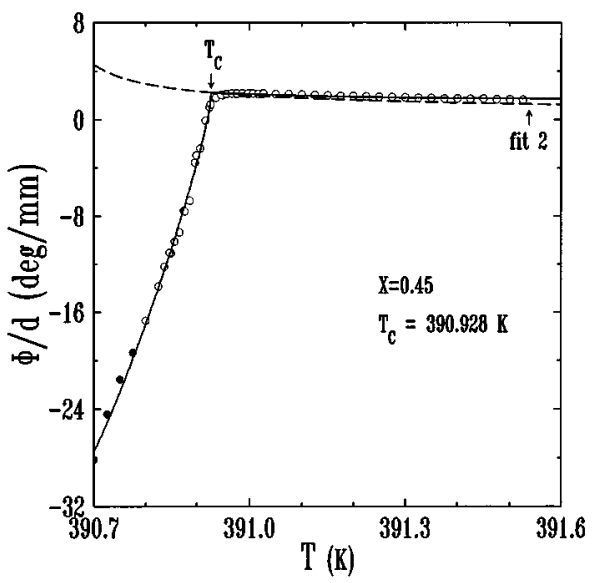

FIG. 8. Comparison of $\Phi / d$ (optical activity) data for $X=0.45$ with a theoretical fit curve based on Eq. (9). The smooth curve represents fit 2 in Table III for which the critical exponent $z=0.52$. The variation of $F_{\text {background }}(T)$ is shown by the dashed curve. The four data points denoted by filled circles were excluded from the fit. 
space for which Fisher renormalization will occur [21]. The heat capacity $C_{p}$ and order parameter $\langle\varphi\rangle$ scale in the following ways:

$$
\begin{gathered}
\Delta C_{p} \sim|\tau|^{-\gamma / \Delta}, \quad\langle\varphi\rangle \sim|\tau|^{\beta / \Delta} \quad(\text { region } 1), \\
\Delta C_{p} \sim|\tau|^{-\gamma}, \quad\langle\varphi\rangle \sim|\tau|^{\beta} \quad(\text { region } 2) \\
\left.\Delta C_{p} \sim|\tau|^{-\gamma /(1-\alpha)}, \quad\langle\varphi\rangle \sim|\tau|^{\beta /(1-\alpha)} \quad \text { (Fisher renorm. }\right),
\end{gathered}
$$

where $\Delta C_{p} \equiv C_{p}-\left[B_{r}+E\left(T-T_{c}\right)\right]$ and $\beta / \Delta=1 / \delta$. Ising values of these exponents are $\beta=0.325, \gamma=1.241, \beta / \Delta=0.208$, $\gamma / \Delta=0.7925, \beta /(1-\alpha)=0.365, \gamma /(1-\alpha)=1.394$, and meanfield values are $\beta=0.5, \gamma=1, \beta / \Delta=0.333, \gamma / \Delta=0.667, \beta /(1$ $-\alpha)=\beta=0.5$, and $\gamma /(1-\alpha)=\gamma=1$.

Although it is theoretically expected that asymptotically close to the critical point, the $X=$ const path should lie in region $1[10,22]$, this choice of path does not agree with the experimental exponents $x$ and $z$ for either Ising or mean-field behavior. It is possible, however, for the $X=$ const path to lie in region 2 over the range of accessible reduced temperatures $\left(\left|\tau_{\min }\right|\right.$ is approximately $2 \times 10^{-4}$ for $C_{p}$ and $\sim 10^{-4}$ for $\left.\langle\varphi\rangle\right)$. In this case, the results from our analysis agree with meanfield theory: $x=\gamma=1.0$ and $z=\beta=0.5$.

Additional support for mean-field (MF) behavior comes from light scattering intensity data on $S, S$-MBBPC and $S, S$ $\mathrm{MBBPC}+\overline{7} \mathrm{~S} 5$ mixtures [23]. Both supercritical pure $S, S$ MBBPC and a mixture with 40 wt. \% MBBPC that may be close to critical show that the intensity $I$ of $m= \pm 2$ mode scattering in the isotropic phase can be described by $I-I_{0}=K /\left(T-T^{*}\right)$, where $I_{0}$ and $K$ are constants and $T^{*}$ is an effective divergence temperature. In the limit $\tau=\left(T-T_{c}\right) / T_{c} \rightarrow 0$, this empirical expression is consistent with theory [10] if (a) $I$ is dominated by the quadratic $\langle\varphi\rangle^{2}$ contribution, i.e., $I=I_{c}-B_{I}^{ \pm}\langle\varphi\rangle^{2}$, and (b) $\langle\varphi\rangle \sim \mid \tau^{1 / 2}$. Thus intensity data also suggest a path in region 2 with $\beta=\beta_{\mathrm{MF}}=0.5$.

It is proposed that the observation of mean-field rather than Ising (or any other) fluctuation behavior is due to long bare correlation lengths associated with this BP III-I critical point. This would lead to a very small critical region, i.e., a small $|\tau|_{c}$ value for crossover from mean-field to critical behavior, just as in the case of Sm- $A-\mathrm{Sm}-C$ transitions [24]. Unfortunately, the Ginzburg $\mid \tau_{c}$ value cannot be calculated, since correlation length data are not available. A light scattering experiment, analogous to x-ray scattering near the Sm$A-\mathrm{Sm}-C$ transition, might permit a test of this suggestion. Indeed, a recent light scattering study of COC has yielded a bare correlation length $\xi_{0}$ of $15 \AA$ associated with BP III short-range correlations near the BP III-I transition [8]. Assumption of a comparable $\xi_{0}$ value for the present system would be consistent with the observed mean-field behavior. It should be noted that mean-field behavior is also observed for the heat capacity and order parameter near the Sm-CSm- $I$ (tilted hexatic) critical point [14]. The path $X=X_{c}$ lies in region 2 for that system as well. Indeed, the theoretical fit to $C_{p}$ data for a near-critical mixture of 8SI+8OSI (methylbutylphenyl octylbiphenyl-carboxylate and its octyloxybiphenyl analog) is almost identical to fit $3 B$ in Table II, except for the amplitudes $A_{1}^{+}$and $A_{2}^{+}$and the sign of $\Delta B$ (see fit 12 in Ref. [14]).

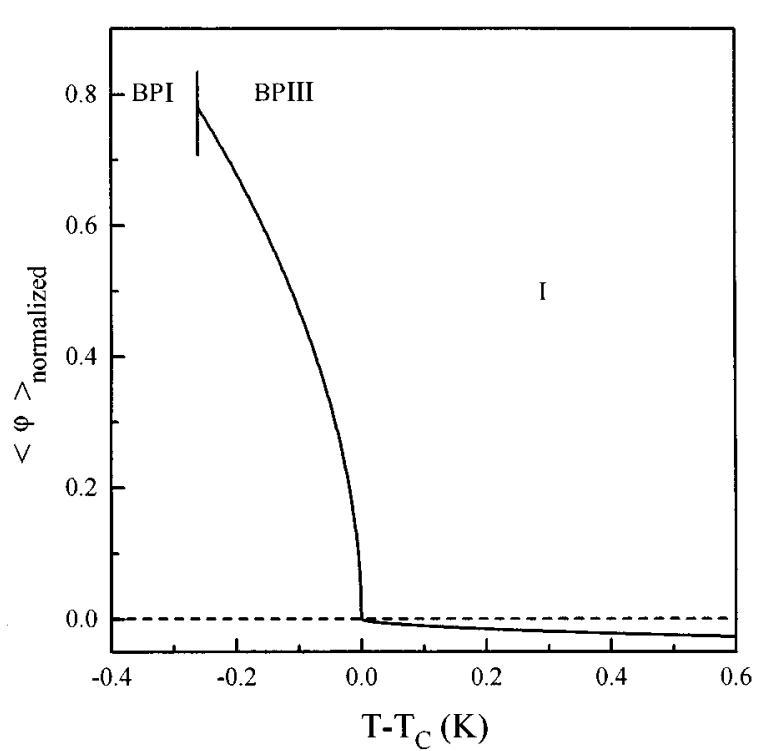

FIG. 9. Plot of the normalized order parameter $\langle\varphi\rangle=\langle\psi\rangle-\langle\psi\rangle_{c}$ versus $T-T_{c}$ for the near-critical mixture with $X=0.45$.

In order to display clearly the temperature variation of the mean-field order parameter near the BP III-I critical point, Fig. 9 shows a plot of $\langle\varphi\rangle$. The quantity shown has been normalized so that $\langle\varphi\rangle\left(T-T_{c}=-0.4 \mathrm{~K}\right)-\langle\varphi\rangle\left(T-T_{c}=+0.6\right.$ $\mathrm{K}) \simeq 1$.0.

\section{Conclusion}

Measurements of $C_{p}$ and $\Phi / d$ on mixtures of $S, S$ $\mathrm{MBBPC}+$ its racemate have shown a change from a firstorder BP III $-I$ transition at low chirality to a critical point at $X_{\mathrm{MBBPC}} \simeq 0.45$ to supercritical behavior for $X \geqslant 0.50$. Analysis of data for the $X=0.45$ mixture indicates that $\Delta C_{p}$ and $\Phi / d$ are consistent with mean-field behavior. Data for $T<T_{c}$ are limited due to the short BP III range; $T(\mathrm{BP}$ I-BP III $)-T_{c}$ (BP III $\left.-I\right)=-0.27 \mathrm{~K}$. It would be desirable to study the BP III-I critical point in a system with an appreciably wider BP III range. It may also be attractive to study a mixture of $S, S$-MBBPC + a racemate consisting of only $R, R$ and $S, S$ enantiomers. Such a mixture is a twocomponent system unlike the present case, which is formally a four-component system. Static and dynamic lightscattering measurements on a critical mixture, like those carried out on pure $S, S$-MBBPC [4], would yield valuable information on the scattered intensity $I$ and the relaxation time for fluctuations of the chiral modes. Structural information about the BP III phase (from optical diffraction or NMR) would be experimental information of obviously great value. Quasielastic light scattering should be able to characterize the correlation lengths near the critical point. Such data would allow a calculation of the Ginzburg critical crossover temperature $\mid \tau_{c}$ and hopefully validate our suggestion that mean-field behavior is seen since $|\tau|>\mid \tau_{c}$ over the accessible $\tau$ range. Finally, it should be noted that in the past the behavior of $\Phi / d$ in a near-critical region has been interpreted in terms of a contribution from the $m= \pm 2$ mode in addition to the $m= \pm 1$ contribution ( $F_{\text {background }}$ ) that is dominant in the isotropic phase [19], and a reexamination of that analysis seems appropriate in light of the present results and current theory [10]. 


\section{ACKNOWLEDGMENTS}

We wish to thank P. H. Keyes and T. Lubensky for helpful discussions and especially $\mathrm{T}$. Lubensky for providing a copy of the theory cited in Ref. [10] prior to its publication.
This work was supported by the National Science Foundation under Grants No. DMR-9224617 and No. DMR9311853. One of the authors (Z.K.) wishes to acknowledge support by the Fulbright program.
[1] Recent reviews include D. C. Wright and N. D. Mermin, Rev. Mod. Phys. 61, 385 (1989); P. P. Crooker, Liq. Cryst. 5, 751 (1989); R. M. Hornreich and S. Shtrikman, Mol. Cryst. Liq. Cryst. 165, 183 (1989); V. A. Belyakov and V. E. Dmitrienko, Usp. Fiz. Nauk 146, 369 (1986) [Sov. Phys. Usp. 28, 535 (1985)].

[2] G. Strajer, R. Pindak, M. A. Waugh, and J. W. Goodby, Phys. Rev. Lett. 64, 1545 (1990); S. R. Renn and T. C. Lubensky, Phys. Rev. A 38, 2132 (1988).

[3] P. H. Keyes, Phys. Rev. Lett. 65, 436 (1990); R. M. Hornreich, M. Kugler, and S. Shtrikman, ibid. 48, 1404 (1982); L. Longa, W. Fink, and H. R. Trebin, Phys. Rev. E 48, 2296 (1993); L. Longa and H. R. Trebin, Phys. Rev. Lett. 71, 2757 (1993).

[4] Z. Kutnjak, C. W. Garland, J. L. Passmore, and P. J. Collings, Phys. Rev. Lett. 74, 4859 (1995).

[5] J. B. Becker and P. J. Collings, Mol. Cryst. Liq. Cryst. 265, 163 (1995).

[6] J. E. Wyse, J. Ennis, and P. J. Collings, Phys. Rev. Lett. 62, 1045 (1989); J. Ennis, J. E. Wyse, and P. J. Collings, Liq. Cryst. 5, 861 (1989).

[7] G. Voets and W. Van Dael, Liq. Cryst. 14, 617 (1993); G. Voets, Ph.D. thesis, University of Leuven, Belgium, 1992 (unpublished).

[8] P. H. Keyes, Phys. Rev. Lett. 59, 83 (1987); E. P. Koistinen and P. H. Keyes, ibid. 74, 4460 (1995).

[9] P. H. Keyes (private communication).

[10] T. C. Lubensky and H. Stark, Phys. Rev. E 53, 714 (1996).

[11] H. S. Kitzerow and P. P. Crooker, Phys. Rev. Lett. 67, 2151 (1991); R. M. Hornreich, ibid. 67, 2155 (1991).
[12] D. K. Yang and P. P. Crooker, Phys. Rev. A 35, 4419 (1987).

[13] M. B. Bowling, P. J. Collings, C. J. Booth, and J. W. Goodby, Phys. Rev. E 48, 4113 (1993).

[14] H. Yao, T. Chan, and C. W. Garland, Phys. Rev. E 51, 4585 (1995).

[15] K. Ema, T. Uematsu, A. Sugata, and H. Yao, Jpn. J. Appl. Phys. 32, 1846 (1993), and references therein.

[16] J. Thoen, E. Bloemen, and W. Van Dael, J. Chem. Phys. 68, 735 (1978).

[17] K. Ema, H. Yao, I. Kawamura, T. Chan, and C. W. Garland, Phys. Rev. E 47, 1203 (1993); see, also, C. W. Garland, Thermochim. Acta 88, 127 (1985); C. W. Garland, Liquid Crystals: Physical Properties and Phase Transitions, edited by S. Kumar (Cambridge University Press, Cambridge England, in press), Chap. 6.

[18] J. Thoen, Phys. Rev. A 37, 1754 (1988); J. Thoen (private communication).

[19] P. R. Battle, J. D. Miller, and P. J. Collings, Phys. Rev. A 36, 369 (1987).

[20] D. Bensimon, E. Domany, and S. Shtrikman, Phys. Rev. A 28, 427 (1983).

[21] X. Wen, C. W. Garland, R. Shashidhar, and P. Barois, Phys. Rev. B 45, 5131 (1992).

[22] P. M. Chaikan and T. C. Lubensky, Principles of Condensed Matter Physics (Cambridge University Press, New York, 1995), Sec. 4.4.

[23] J. E. Wyse and P. J. Collings, Phys. Rev. A 45, 2449 (1992).

[24] R. J. Birgeneau, C. W. Garland, A. R. Kortan, J. D. Litster, M. Meichle, B. M. Ocko, C. Rosenblatt, L. J. Yu, and J. Goodby, Phys. Rev. A 27, 1251 (1983). 